\title{
12-LEAD ECG TRAINING: THE WAY FORWARD
}

\author{
Corresponding author: \\ Guillaume Alinier, MPhys, PGCE, CPhys, MInstP, ILTM: \\ Hertfordshire Intensive Care and Emergency Simulation Centre Co-ordinator \\ Faculty of Health \& Human Sciences \\ University of Hertfordshire \\ College Lane \\ Hatfield \\ Hertfordshire, AL10 9AB \\ Tel: $+44(0) 1707-286395$ \\ Fax: $+44(0) 1707-284199$ \\ Email: G.Alinier@herts.ac.uk
}

Ray Gordon, BSc, CEng, MIEE, MIPEM: Associate Head

School of Electronic, Communication and Electrical Engineering

University of Hertfordshire

College Lane

Hatfield

Hertfordshire, AL10 9AB

Tel: $+44(0) 1707-284187$

Fax: +44 (0)1707-284199

Email: R.Gordon@herts.ac.uk

Colin Harwood, BA, RMN, SRN, ILTM: Principal Lecturer

School of Paramedic Sciences, Physiotherapy and Radiography

University of Hertfordshire

College Lane

Hatfield

Hertfordshire, AL10 9AB

Tel: $+44(0) 1707-285923$

Fax: +44 (0)1707-285904

Email: C.Harwood@herts.ac.uk

William B Hunt, BPharm, PhD, MRPharmS: Associate Dean

Faculty of Health \& Human Sciences

University of Hertfordshire

College Lane

Hatfield

Hertfordshire, AL10 9AB

Tel: $+44(0) 1707-285910$

Fax: +44 (0)1707-285904

Email:W.B.Hunt@herts.ac.uk 


\section{ABSTRACT}

The teaching of electrocardiography (ECG) monitoring has not changed for decades and still very much relies on access to real patients for practice. However there is nowadays an even greater need for modern training tools in this field as more healthcare professionals than ever before need to be trained on 12-lead ECG monitoring techniques. For example in many parts of the country ambulance paramedics use features observed from 12-lead ECG monitoring equipment to determine whether or not a patient can receive pre-hospital thrombolytic therapy. Because important decisions are made without the presence of a cardiologist, it is essential that the training is carried out with the most realistic tools, including tools which give realistic feedback of the consequences of incorrect electrode replacement. Current mannequins for ECG training are designed with protruding electrodes on the chest, which act as cues for trainees. There is therefore a need for a realistic simulation training tool to teach 12-lead ECG interpretation which includes the ability to give this feedback. We are currently working on the development of such a training tool and it is expected that it will be of great interest to medical, nursing and paramedic schools.

\section{KEYWORDS}

12-lead, ECG monitoring, training 


\section{INTRODUCTION}

Despite the increased use of high-tech equipment in healthcare education there are a number of areas where these educational approaches need to be enhanced. Indeed for some of the fundamental physiological measurement techniques used clinically there is no robust teaching tool. For example, a clear understanding of ECG monitoring can be difficult to grasp if the appropriate explanations are not given and if it cannot be practised for real. The appreciation of correct electrode positioning may not be recognised by some students. They may think that as long as each electrode has a good surface contact with the skin the expected normal sinus rhythm signal will be clearly detected and displayed on the ECG monitor. In fact the signal received directly depends on the electrode's location as well as on the contact they have with the skin. The electrodes can figuratively be considered as observers or video cameras looking at the electrical activity of the heart. Depending from which direction the heart is "observed", or monitored, the electrical fields produced by the cells' depolarisation will look different (Garcia \& Holtz 2001). This concept is not always very easy to inculcate into people who are trying to learn about ECG monitoring and interpretation. Current training tools do not allow for "free" positioning of electrodes to demonstrate and observe the direct effect lead misplacement may have. Currently, the only option is to use volunteers but this raises a number of issues that will be discussed later. There is a need for an effective and realistic teaching tool to be designed so that this effect can be clearly demonstrated and used for training purposes. This would give trainees the opportunity for safe and extensive training, without ethical concerns or causing any inconvenience to patients. This would benefit and improve the training of healthcare staff who may be called on to deliver thrombolytic drugs, and ultimately to improve patient care. 


\section{THE CURRENT TEACHING OPTIONS}

There is currently a wide range of teaching techniques that can be used to train healthcare professionals in the use of 12-lead ECG monitoring equipment and ECG interpretation (see table 1).

Table 1

The methods identified range from traditional lectures and reading material, to the use of a sophisticated training mannequin or software packages. From the most basic to the most advanced, none of the current teaching methods are ideal. The traditional teaching methods have some disadvantages in terms of patient or volunteer consent and discomfort, but also in terms of transferability of skills and teaching effectiveness. Depending on the students' learning types, principles learnt from lectures or books can sometimes be difficult to apply to real life circumstances. The more advanced teaching methods identified in table 1 make use of simulation which can either be screen-based (computer software) or physical (realistic models or mannequins). The principle of realistic simulation as a learning and teaching tool draws its ideas from the theories of experiential learning (Kolb 1984, Cioffi 2001). Experiential learning comes in many different forms and is about giving students the opportunity to experiment or interact with equipment or procedures in order to develop their capabilities (Graves 1993, p 105). Ideally practising is a means of helping students or trainees to become familiar with a specific technique, protocol or piece of equipment by using it repeatedly in its usual environment. In that case the best way to teach how to position the ECG electrodes would be to do it on a patient. In addition to the discomfort caused by the repeated placement and removal of 
electrodes on a patient or volunteer, ethical issues need to be addressed. These would include the use of patients in life threatening situations, or critically ill, or, in the case of volunteers, the potential for discovering unsuspected cardiovascular problems, or to wrongly diagnose a pathology that they do not have, causing them unnecessary stress. In addition, while trainees may be able to see the consequences of incorrect electrode position with normal ECG's, as obtained from volunteers, it is much more difficult to arrange for them to see what these consequences might be in cases of specific abnormalities. There are a number of ECG simulators on the market that can be connected to an ECG machine. They are in fact ECG signal generators presented as a box and very suitable to test ECG machines. Manufacturers generally also offer models of ECG simulators that are internally connected to a mannequin to simulate its ECG output. Some of the major advantages over software ECG training packages are that they allow for the palpation of physical landmarks by candidates and generally enable real ECG monitoring equipment to be connected on them. However, until now the technology used is such that mannequins have apparent chest connectors, hence limit the possibilities of positioning errors by trainees.

\section{BACKGROUND INFORMATION}

Our simulation centre was opened in 1998 and provides a realistic clinical environment simulating a 3-adult bed Intensive Care Unit with paediatric and neonatal facilities. Work has been ongoing on the development of technological aspects of medical simulation to facilitate teaching. The centre is equipped with a range of sophisticated adult patient simulators, and other mannequins and part-task trainers such as airway management or cannulation models. Funding was provided for 3 years by the British Heart Foundation (BHF Project number: Edcomm/Oct98/9d) to investigate the effect of simulation training in the retention of knowledge and 
nursing acute care skills using Objective Structured Clinical Examinations (Series of short practical and theoretical tests) as a comparative assessment tool (Alinier et al 2004). The combination of our increasing activity in the field of medical simulation training and our internal growing demand for 12-lead ECG teaching, primarily for paramedic educational requirements, has lead us to consider the development of a new training tool, as presented later in this paper.

\section{THE NEED FOR 12-LEAD ECG}

Coronary Heart Disease (CHD) caused over 135,000 deaths in the UK in 1998 (Peterson et al 2000). Each minute is critical for a patient with CHD or complaining of chest pain. Rapid and accurate diagnosis of such patients is of utmost importance and can only be achieved if staff receive adequate training. 12-lead ECG is a key investigation element for the diagnosis of $\mathrm{CHD}$. Healthcare practitioners need to be trained in the appropriate use of this monitoring technique and in the delivery of thrombolytic therapy. It includes the placement of up to 10 electrodes on a patient's chest at precise locations and the interpretation of the traces captured to confirm or refute the diagnosis and the need for thrombolysis. For this reason there is a national increase in the use of 12-lead ECG in the hospital and pre-hospital setting. Research has already shown that accurate and early diagnosis of a myocardial infarction can be achieved by a trained paramedic, and this can be a basis for earlier thrombolysis (Furber et al 1996). The speed and accuracy of diagnosis required demands a high level of confidence and skill on the part of the health professional involved. There is currently no other option but to train on real patients, which is where mistakes are not allowed as they can cost their lives. High quality, sophisticated teaching tools are required to achieve this level of skill. 
The new guidelines of the National Service Framework (NSF) for CHD (DoH 2000) emphasise the need to improve the current healthcare provision system. The UK Government is committed to building a new NHS to reduce inequalities, improve health, and raise the quality of care (DoH 2000). A significant part of the proposed 10-year plan is to reduce premature death from CHD in UK. To meet this aim, the prevention, diagnosis and treatment of CHD is being revised. A European study showed that pre-hospital thrombolysis by paramedics could reduce short-term mortality by $16 \%$ (Boissel 1995). Taking into consideration that around $12 \%$ of paramedic journeys involve patients with cardiac chest pain and that $4 \%$ of them would qualify for pre-hospital thrombolysis (Weaver et al 1990, Weaver et al 1993), the need to implement new treatment protocols becomes even more urgent. As a result ambulance and A\&E services are expected to help reduce the call-to-needle time for thrombolysis at a national level by improving response times and by administrating thrombolytic drugs when appropriate. To that effect the proficient use of 12-lead ECG monitoring technique will soon become a requirement for over 8500 registered paramedics (CPSM 2001) and part of the 170,000 qualified nurse workforce (DoH 2001) in order to deliver care related to myocardial infarctions or other chest pain related conditions, thereby improving the standard of care delivered to patients. Nursing and paramedic staff will therefore be required to assess whether a patient can be treated with thrombolytic drugs in case of a suspected cardiac problem. Part of this assessment relies on the use of a 12-lead ECG to confirm or refute the diagnosis. The assessment criteria used to determine whether or not thrombolysis is carried out include a number of features observable from an ECG such as abnormal ST elevation, QRS width, and atrioventricular block (JRCALC 2001, EMAS 2002). Important clinical decisions will be made upon the basis of these assessments, and whether or not the ECG was performed correctly is crucial. 
Key to the successful implementation of this programme will be the quality of the staff delivering the treatment. This will depend to a great extent on the appropriateness of nurses, paramedics and A\&E staff, as well as on the training they receive. The executive summary clearly specifies that "those responsible for education will be expected to provide opportunities which help put the NSF into practice" (DoH 2000, p. 11). Errors made in taking the ECG, such as the misplacement of electrodes can cause incorrect interpretation, misdiagnosis, and subsequent improper treatment of the patient (Heden et al 1995, Pastor et al 2001). Educational processes and tools which permit students to gain a high level of competence in this area are therefore vital. However, as it has already been highlighted, there is currently no adequate training tool to help the acquisition of these skills as one comprehensive package. Current full body simulators rely mainly on the intervention of the trainer to assess the action taken by the students and make the appropriate change, and screen-based simulators do not offer the required level of realism needed to acquire the physical examination skills to appropriately locate the ECG electrodes. There is therefore a need for an efficient training tool to replace the use of real persons to teach 12-lead ECG monitoring techniques. The ideal teaching tool for the placement of ECG electrodes would consist of a realistic mannequin generating ECG waveform according to the positioning of each electrode.

Picture 1

\section{PROPOSED 12-LEAD ECG TRAINING MODEL}

Our current research aims at developing an accurate and realistic training mannequin that will allow for electrodes to be placed anywhere on its chest, provide a 
corresponding 12-lead ECG trace on a real patient monitor, be able to present a range of cardiac pathologies, and to respond to drug administration such as thrombolytic therapy. The system would have to be able to recognise each of the 10 leads attached to the mannequin so that, for example, inversion of any of the leads would result in an inverted signal on the ECG monitor. Ideally the training system could be used with any type of ECG monitoring equipment with up to 10 physical leads, from which the 12-lead ECG traces are obtained. The main novelty will be that the chest of the mannequin, which will have to be very realistic and include all anatomical landmarks, will need to allow the system to recognise the electrodes' position without requiring apparent chest connectors already in place (Picture 1). It would oblige the students to think where they should stick the electrodes and the corresponding ECG leads. This coupled with the fact that a corresponding and accurate 12-lead ECG will have to be displayed, corresponding to the degree and nature of electrode misplacement will be the major technological challenges of this project. Although much basic work has been done, the precise different approaches that can be adopted to develop a software model to simulate realistic results are still being investigated. Modelling of the effect of thrombolytic drugs on 12-lead ECG traces also needs to be researched and developed. The ultimate product of this project will be an interactive 'model-driven simulation' tool which reacts in a realistic way to both correct and erroneous students actions in ECG assessment. Such a development would be valuable in training students to safely apply and practise their skills. It could be used without interruption, as, contrarily to a patient or a volunteer, the mannequin could cope with being used continuously and in turn by large numbers of students. A mannequin that enables ECG traces to be observed on a monitor according to the actual position of the electrodes would certainly enable students to become more familiar with placement of electrodes for 12-lead ECG monitoring, the recognition of dysrhythmia or common monitoring problems. 


\section{DISCUSSION}

There is a real interest in trying to develop effective teaching methods related to the use and interpretation of ECG (Bowler 1996) but the development of such technology without specific support is a permanent challenge and in our case has until now relied on the motivation of the team members, the inter-Faculty collaboration, and the cooperation with other institutions. Over the past few years, 12-lead ECG has increasingly become a standard clinical examination procedure, and is being used in a greater number of healthcare disciplines. It proves to be a very valuable piece of monitoring equipment when it comes to diagnose heart problems, but it also proves more complex to use than a traditional 3 or 4-lead ECG, normally used to simply monitor the regularity and pace of the heart. In the near future all UK ambulance services will have to replace their current $3-4$ lead monitoring systems with 12-lead ones, which means that a great number of staff, as quantified earlier in this paper, will require appropriate training. This need has been identified because paramedic 12-lead ECG diagnosis helps in reducing the delay times before the injection of thrombolysis by permitting earlier identification of victims of Myocardial Infarction (MI) and enabling their admission directly to a coronary care unit (Furber et al 1996).

It is expected that the closer to the real thing the training medium or material can be, the more profitable the experience is for the student. Developing a system that tries to replicate a particular function of a human being is rarely straightforward. One of the two key challenges of this project, which are namely the recognition by the system of the exact position of the electrodes and the development of a cardiac model to produce a corresponding 12-lead ECG trace, has been solved. Another important aspect of the development of a modern and realistic ECG training tool is that its shape and forms actually need to resemble a real patient rather than a very fit 
person. The skills acquired when learning how to position ECG electrodes on a fit patient simulator are not always readily transferable to an obese or female patient. Developing an adaptable ECG training system would greatly enhance its usefulness. It is greatly hoped that our project will be taken further in the years to come in order to benefit healthcare professionals and ultimately, the patient community.

\section{CONCLUSION}

Although thrombolytic drugs appropriately administered can save a life, judgemental error or misdiagnosis due to a poor ECG trace or misinterpretation could have a lifethreatening outcome for the patient. The development of a new ECG training model would help health care staff to acquire important skills that would contribute to the saving of life. The medical training device that we are proposing to develop has long been wanted by clinical trainers and trainees as there is currently no adequate training tool to teach 12-lead ECG acquisition and interpretation. The University research team has found a solution to the first challenge of this project which is the detection of electrodes' positions and built a prototype that needs to be further developed. The next step is the software development of the project to display the corresponding ECG. The system described above would allow users to fully explore and understand the principles of 12-lead ECG without causing discomfort to patients or putting them at risk. With or without expert supervision, it could enable trainees to appreciate the importance of correctly placing the electrodes on the patient's chest and observe the effect on the ECG traces. This would enable more staff to be trained on the use and interpretation of 12-lead ECG, as well as on the appropriate administration of thrombolytic drugs. Patients would benefit by being cared for by better prepared healthcare professionals and receive earlier treatment. 


\section{ACKNOWLEDGEMENTS}

The authors of the article would like to thank Mr Anthony Herbland, from the School of Electronic, Communication and Electrical Engineering at the University of Hertfordshire for his technical advice on the design of the hardware prototype, as well as placement students from the Department of Physical Measurements, Institut Universitaire Technologique of Evreux, which is part of the University of Rouen (France) for their work on the project. We are also very grateful to Mr Bryan McClure, from the department of Marketing and Communications, for his perseverant support and encouragements to carry on working on this project.

\section{$\underline{\text { REFERENCES }}$}

Alinier G, Hunt WB, Gordon R, 2004. Determining the effect of realistic simulation in nurse education: Study design and initial results. Nurse Education in Practice 4(3), 200-207

Boissel, J.P., 1995. The European Myocardial Infarction Project: an assessment of pre-hospital thrombolysis. International Journal of Cardiology. 49, S29-S37

Bowler, G., 1996. ECG and arrhythmias during resuscitation: a new educational package from the resuscitation council (UK). Resuscitation 31(3), 34-34

Cioffi, J., 2001. Clinical simulations: development and validation. Nurse Education Today 21(6), 477-486 
Council for Professions Supplementary to Medicine. Annual Report 2001-2002. Park House, 184 Kennington Park Road, SE11 4BU. London

Department of Health, 2001. NHS Hospital and Community Health Services. NonMedical Workforce Census - England: 30 September 2001. Table 1.5b, p9. ISBN 1 $84182495 X$

Department of Health, 2000. National Service Framework for Coronary Heart Disease. Executive Summary.

(http://www.dh.gov.uk/assetRoot/04/05/75/25/04057525.pdf) accessed on the $6 / 07 / 05$

East Midland Ambulance Service NHS Trust, 2002. A comparison Review of PreHospital Thrombolysis Pilot Times in a Rural/Urban Area of the East Midlands. Published on the website of The National Clinical Effectiveness Programme of the Ambulance Service Association (http://www.asancep.org.uk/PHTTIMEA.pdf) accessed on the 6/07/05

Furber, R., Thomas, B., Joy, A., Adamowicz, M., Millar-Craig, M., 1996. Paramedic 12-lead ECG diagnosis with direct admission to coronary care results in reduction in delay to thrombolysis. Resuscitation 31(3), 20-20

Garcia, T.B., Holtz, N.E., 2001. 12-lead ECG : The Art of Interpretation. Jones \& Bartlett Publishers, London.

Graves N, 1993. Learner-managed-learning: Practice, Theory and Policy. Leeds: Higher Education for Capability/World Education Fellowship 
Heden, B., Ohlson, M., Edenbrandt, L., Rittner, R., Pahlm, O., Peterson, C., 1995.

Artificial Neural Networks for recognition of Electrocardiographic Lead Reversal.

American Journal of Cardiology 75 (14), 929-933

Joint Royal Colleges Ambulance Liaison Committee, 2001. National Clinical Audit of AMI including prehospital thrombolysis. Published on the website of The National Clinical Effectiveness Programme of the Ambulance Service Association (http://www.asancep.org.uk/ami.htm) accessed on the 6/07/05

Kolb, D., 1984. Experiential Learning: Experience as the Source of Learning and Development. , Prentice-Hall, Englewood Cliffs, NJ

Peterson, S., Press, V., Rayner, M., 2000. Coronary Heart Disease Statistics. British Heart Foundation Database. London

Pastor, J.A., Castellanos, A.C., Myerburg, R.J., Pallares, D.S., 2001. Apparent misplacement of chest electrode on left leg: a unique example of electrodal confusion. American Journal of Cardiology 88 (7), 829-830

Weaver, W.D., Cerqueira, M., Hallstrom, A.P., Litwin, P.E., Martin, J.S., Kudenchuk, P.J., Eisenberg, M., 1993. Prehospital initiated versus hospital-initiated thrombolytic therapy: The Myocardial Infarction Triage and Intervention Trial. Journal of American Medical Association 270 (10), 1211-1216

Weaver, W.D., Eisenberg, M.S., Martin, J.S., Litwin, P.E., Schaeffer, S.M., Ho, M.T., Kudenchuk, P., Hallstrom, A.P., Cerqueira, M.D., Copass, M.K., Kennedy, J.W., Cobb, L.A., Ritchie, J.L., 1990. Myocardial Infarction Triage and Intervention (MITI) 
Project Group - Phase 1: Patient characteristics and feasibility of prehospital initiation of thrombolytic therapy. Journal of American College of Cardiology 15 (5), 925-931 


\begin{tabular}{l|l}
\multicolumn{1}{c|}{ Traditional teaching methods } & \multicolumn{1}{|c}{ Advanced teaching methods } \\
\hline - Books & - PC based ECG software packages \\
- Lectures & - 12 lead ECG signal generators \\
- Videos & - Advanced training mannequins (Patient \\
- Hospital placements (using patients) & simulators) \\
- Practical laboratory sessions (using & \\
volunteers) &
\end{tabular}

Table 1: List of current methods for teaching ECG lead placement and ECG interpretation.

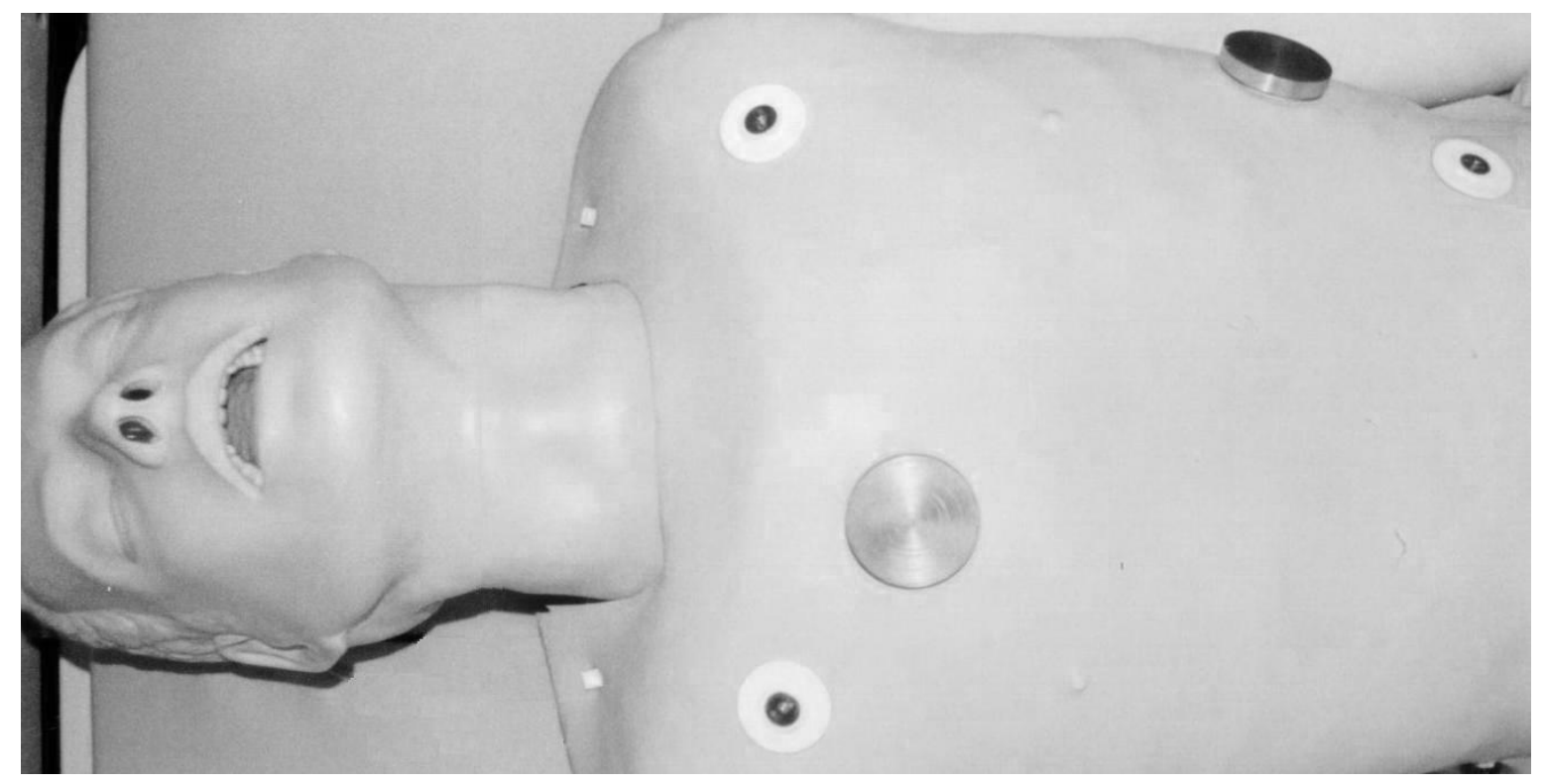

Picture 1: Commercially available Advanced Life Support training mannequin with apparent electrode connectors on the chest. 